\title{
BMJ Open Statistical methods for non-adherence in non-inferiority trials: useful and used? A systematic review
}

\author{
Matthew Dodd (D) , ${ }^{1,2}$ Katherine Fielding (D) , 3 James R Carpenter (D) ,1,4 \\ Jennifer A Thompson (D), , Diana Elbourne (D) ${ }^{1,2}$
}

To cite: Dodd M, Fielding K, Carpenter JR, et al.

Statistical methods for nonadherence in non-inferiority trials: useful and used? A systematic review. BMJ Open 2022;12:e052656. doi:10.1136/ bmjopen-2021-052656

- Prepublication history and additional supplemental material for this paper are available online. To view these files, please visit the journal online (http://dx.doi.org/10.1136/ bmjopen-2021-052656)

Received 21 April 2021 Accepted 16 December 2021

Check for updates

(C) Author(s) (or their employer(s)) 2022. Re-use permitted under CC BY-NC. No commercial re-use. See rights and permissions. Published by BMJ.

${ }^{1}$ Department of Medical Statistics, London School of Hygiene \& Tropical Medicine, London, UK

${ }^{2}$ Clinical Trials Unit, London

School of Hygiene \& Tropical

Medicine, London, UK

${ }^{3}$ Department of Infectious

Disease Epidemiology, London

School of Hygiene \& Tropical

Medicine, London, UK

${ }^{4}$ MRC Clinical Trials Unit, UCL, London, UK

Correspondence to

Matthew Dodd;

matthew.dodd@Ishtm.ac.uk

\section{ABSTRACT}

Background In non-inferiority trials with non-adherence to interventions (or non-compliance), intention-to-treat and per-protocol analyses are often performed; however, nonrandom non-adherence generally biases these estimates of efficacy.

Objective To identify statistical methods that adjust for the impact of non-adherence and thus estimate the causal effects of experimental interventions in non-inferiority trials.

Design A systematic review was conducted by searching the Ovid MEDLINE database (31 December 2020) to identify (1) randomised trials with a primary analysis for non-inferiority that applied (or planned to apply) statistical methods to account for the impact of non-adherence to interventions, and (2) methodology papers that described such statistical methods and included a non-inferiority trial application.

Outcomes The statistical methods identified, their impacts on non-inferiority conclusions, and their advantages/disadvantages.

Results A total of 24 papers were included (4 protocols, 13 results papers and 7 methodology papers) reporting relevant methods on 26 occasions. The most common were instrumental variable approaches $(n=9)$, including observed adherence as a covariate within a regression model $(n=3)$, and modelling adherence as a time-varying covariate in a time-to-event analysis $(n=3)$. Other methods included rank preserving structural failure time models and inverse-probability-of-treatment weighting. The methods identified in protocols and results papers were more commonly specified as sensitivity analyses $(n=13)$ than primary analyses $(n=3)$. Twelve results papers included an alternative analysis of the same outcome; conclusions regarding non-inferiority were in agreement on six occasions and could not be compared on six occasions (different measures of effect or results not provided in full).

Conclusions Available statistical methods which attempt to account for the impact of non-adherence to interventions were used infrequently. Therefore, firm inferences about their influence on non-inferiority conclusions could not be drawn. Since intention-to-treat and per-protocol analyses do not guarantee unbiased conclusions regarding non-inferiority, the methods identified should be considered for use in sensitivity analyses.

PROSPERO registration number CRD42020177458.

\section{Strengths and limitations of this study}

- This is the first systematic review to identify statistical methods that attempt to account for the impact of non-adherence to interventions in randomised non-inferiority trials.

- A description and critique of the statistical methods identified is provided, along with their target estimands.

- Publications from any year, journal or disease area/ patient population were reviewed independently by two authors.

- One author extracted the data from the eligible papers.

- While statistical analysis plans were requested for eligible trials, these could not be obtained for all included trials.

\section{INTRODUCTION}

Non-inferiority trials, which assess whether a new intervention is not worse than a proven comparator by more than a clinically acceptable amount, are becoming increasingly common. ${ }^{1-3}$ They are principally used when it is hoped that the new intervention may convey some advantage other than better efficacy (its effect under ideal conditions), such as improved safety, tolerability, convenience or reduced cost. ${ }^{45}$

One of the challenges in these studies, and the focus of this review, is how participants not receiving their randomly assigned intervention according to the trial protocol (termed non-adherence or non-compliance) should be handled in the statistical analysis. ${ }^{6}$ Examples of non-adherence include not receiving a surgical intervention as planned, not taking all of the prescribed doses of a medication, or not attending all of the sessions of an exercise rehabilitation programme. Such nonadherence is common in trials and has been associated with poorer health outcomes. ${ }^{7-9}$ It can bias estimates of efficacy in either direction and so obtaining an accurate and reliable measure of adherence and accounting for any 
non-adherence in the statistical analysis of these studies is essential. ${ }^{10}{ }^{11}$ Non-adherence may also be linked with missing outcome data if, for example, the trial protocol stipulates that further follow-up is no longer required once adherence drops below a specific threshold or if non-adherent participants become lost to follow-up. The terms adherence and compliance are often used interchangeably, though adherence is preferred here since it is felt to better reflect the partnership between the healthcare provider and participant.

A simple approach to handling non-adherence is to define and analyse different analysis sets based on participants' observed levels of adherence, with consistent results providing greater confidence in the trial conclusions. ${ }^{1}$ In the setting of non-inferiority trials, the intention-to-treat (ITT) and per-protocol (PP) populations have been advocated and are commonly used. ${ }^{412}{ }^{13}$ However, agreement between the ITT and PP results of these trials does not guarantee that conclusions regarding non-inferiority are free from bias caused by differential, or non-random, nonadherence (where the factors leading to non-adherence are associated with outcomes). ${ }^{14-16}$

Standard ITT analyses typically include all participants in their randomised groups irrespective of the intervention actually received. ${ }^{17}$ Thus, they reflect the effect of assigning individuals to interventions in clinical practice where not everyone is fully adherent (also known as the 'effectiveness' of an intervention). This approach preserves the balance in known and unknown prognostic factors afforded by randomisation and so any difference in outcomes between study arms can be attributed solely to the experimental intervention. ${ }^{18}$ However, in the presence of non-adherence, ITT analyses may yield biased estimates of efficacy (also known as the 'causal effect' of an intervention). ${ }^{19}$ In non-inferiority trials, where efficacy and effectiveness may be considered equally important, this can increase the probability of falsely claiming non-inferiority and, therefore, accepting a worse intervention. $^{11}$

Modified ITT (mITT) analyses are commonly used to address some of the limitations with standard ITT methods. ${ }^{20}$ This approach allows some randomised participants, such as those who never receive any of the allocated intervention or who are identified as ineligible after randomisation, to be excluded according to prespecified rules. ${ }^{18}$ However, across trials, there is substantial variability in how this population is defined and bias may be introduced by subjectively excluding individuals from analysis. ${ }^{18}{ }^{20}$ In addition, mITT analyses are not typically used to account for the impact of non-adherence.

PP analyses estimate the efficacy of interventions typically by excluding or censoring individuals with major protocol violations, including those who are nonadherent to their allocated intervention. ${ }^{16}{ }^{17}$ Excluding participants in this way can lead to selection bias because non-adherent individuals generally differ from those who are fully adherent with respect to prognostic factors. ${ }^{21} 22$ Furthermore, using a PP analysis to address differential non-adherence is likely to reduce the protection provided by randomisation, so that trial arms are not fully comparable; this potentially biases the study results in either direction. ${ }^{11}$ In other words, any difference in outcomes between trial arms may no longer be due to the experimental intervention only. To obtain valid results from a PP analysis, we need to recover the protection due to randomisation, typically through a statistical method that (given certain assumptions) correctly adjusts for factors associated with both adherence and outcome (confounders). ${ }^{21}$

Statistical techniques that attempt to account for the impact of non-adherence and thus estimate the causal effects of experimental interventions exist. These range from simple approaches, such as including observed adherence as a covariate within a regression model, which like PP analyses is susceptible to selection bias, to more sophisticated techniques, such as instrumental variable (IV) methods and inverse-probability weighting, which allow for non-adherence while attempting to maintain the balance produced by randomisation. ${ }^{23}{ }^{24}$ Several of these methods attempt to estimate the complier average causal effect (CACE), which is the causal effect of an intervention for individuals who would always be fully adherent regardless of assignment (known as compliers). ${ }^{25}$ In other words, it is a comparison of the average outcome among those who are fully adherent in the experimental arm with the average outcome among the comparable group in the control arm who would fully adhere to the experimental intervention, if offered.

It is unclear which of the alternative methods have been applied in the setting of non-inferiority trials, to what extent, and with what results. Therefore, this systematic review aimed to identify statistical methods that can be used to account for the impact of non-adherence to interventions (thereby estimating the causal effects of experimental interventions) in randomised non-inferiority trials. Secondary aims were to quantify the use of such methods in these studies and examine their impact on non-inferiority conclusions.

\section{METHODS}

The Ovid MEDLINE database was searched for terms related to adherence, non-inferiority trials and statistical methods for handling non-adherence in the titles, abstracts and keywords of papers published up to 31 December 2020 (full search strategy is provided in the online supplemental appendix 1). Eligibility based on identifying appropriate statistical methods was assessed using a three-stage process. First, two authors independently reviewed the title and abstract of each paper. Those where the comparison was not randomised, the primary analysis was not for non-inferiority, or the analysis assessed cost-effectiveness were excluded (costeffectiveness analyses were not of interest because the focus of this review was on estimating the efficacy of interventions). Papers not published in English were 
also excluded. If the full text was unavailable, the abstract was reviewed against the eligibility criteria to ensure that key papers were not excluded. Next, an automated search of the full texts was performed in order to identify those containing the terms 'compliance', 'adherence' or 'complier'. Finally, full-text reviews of the remaining papers were performed independently by two authors to identify (1) randomised trials with a primary analysis for non-inferiority that applied (or planned to apply, for protocol papers) statistical methods to account for the impact of non-adherence to interventions, and (2) methodology papers that described such statistical methods and included a non-inferiority trial application. Any discrepancies between reviewer pairs were discussed with a third author in order to reach a consensus. In addition, statisticians within the field were consulted in order to identify key publications, and the reference lists and citations of eligible papers searched for relevant analyses (performed by one author (MD)). Meta-analyses and systematic reviews identified were also searched for eligible noninferiority trials. Where a trial's published protocol and results paper were both eligible and reported the same statistical method of interest, the protocol paper was excluded to avoid double counting. Statistical analysis plans were requested for all eligible trials.

A standardised electronic form was used to extract the relevant information from each paper considered eligible. This included details of the trial characteristics (journal, year of publication, disease area or patient population, unit of randomisation, type of experimental intervention, type of primary outcome and non-inferiority margin), non-adherence to the interventions (definitions and estimated levels of non-adherence), the statistical method attempting to account for non-adherence (name of the method, estimand, estimate of effect and confidence interval (CI), conclusion regarding non-inferiority and any advantages/disadvantages of the method stated) and any other analyses applied to the same outcome (analysis population, estimand, estimate of effect and CI, and conclusion regarding non-inferiority). Data extraction was performed by one author (MD). The primary outcome was the statistical method applied (or planned to be applied) in order to account for non-adherence to the interventions. Other outcomes were the impact of applying these methods on the trial conclusions (compared with other analyses applied to the same outcome, where available) and the advantages and disadvantages of the methods where stated by the authors. The impact of applying the methods of interest was assessed using trial results papers only.

This systematic review was registered with PROSPERO and conducted in accordance with the Preferred Reporting Items for Systematic Reviews and MetaAnalyses statement. ${ }^{26}$ Information was largely combined using a narrative synthesis approach, that is, 'synthesis of findings from multiple studies that relies primarily on the use of words and text to summarise and explain the findings of the synthesis'. ${ }^{27}$ All analyses were conducted using Stata V.15.1.

\section{Patient and public involvement}

Patients or the public were not involved in the design, conduct, reporting, or dissemination plans of our research.

\section{RESULTS}

After removing duplicate publications, our search identified 3235 papers. Of these, 934 were excluded following review of the titles and abstracts, 790 did not contain any keywords in the full texts and 1489 were excluded after full-text review, leaving 22 papers whose citations and reference lists contained a further 5 papers meeting eligibility criteria. After removing publications of the same trial reporting identical statistical methods of interest, 24 papers remained (figure 1).

The 24 publications, which consisted of 4 protocols, 13 results papers and 7 methodology papers, reported relevant methods on 26 occasions (2 methodology papers both contained 2 relevant analyses). Four of the analyses included in methodology papers were re-analyses of non-inferiority trials, one included a simulation study based on a non-inferiority trial and four included simulation studies not based on real trials. Fifteen of the 24 papers included (63\%) were published within the last 5 years and the most common type of experimental intervention studied was drug interventions $(35 \%)$ (table 1; online supplemental table A1).

\section{Non-adherence to interventions}

Non-adherence to the randomly assigned interventions was defined in the methods, statistical analysis plan or results section of most analyses $(n=19,73 \%)$. Fifteen $(79 \%)$ used a binary definition of adherence, whereas $3(16 \%)$ used a continuous measure (one was unclear). Of the 19 analyses that defined non-adherence to the interventions, 13 reported estimates of non-adherence (the remaining 6 were protocols or simulation studies). More than half reported estimates of non-adherence that were no more than $10 \%$, though the range was wide $(1.7 \%-51.3 \%)$ (table 2$)$. For reasons that were not reported, two papers provided data on non-adherence to the interventions in only one arm of the trial.

\section{Statistical methods for handling non-adherence to interventions}

In total, 11 different statistical methods that attempt to account for non-adherence to interventions were identified (table 3). The most common were IV approaches $(n=9,35 \%)$, including observed adherence as a covariate within a regression model $(n=3,12 \%)$, and modelling adherence as a time-varying covariate in a time-to-event analysis $(\mathrm{n}=3,12 \%)$. Other methods included rank preserving structural failure time models 


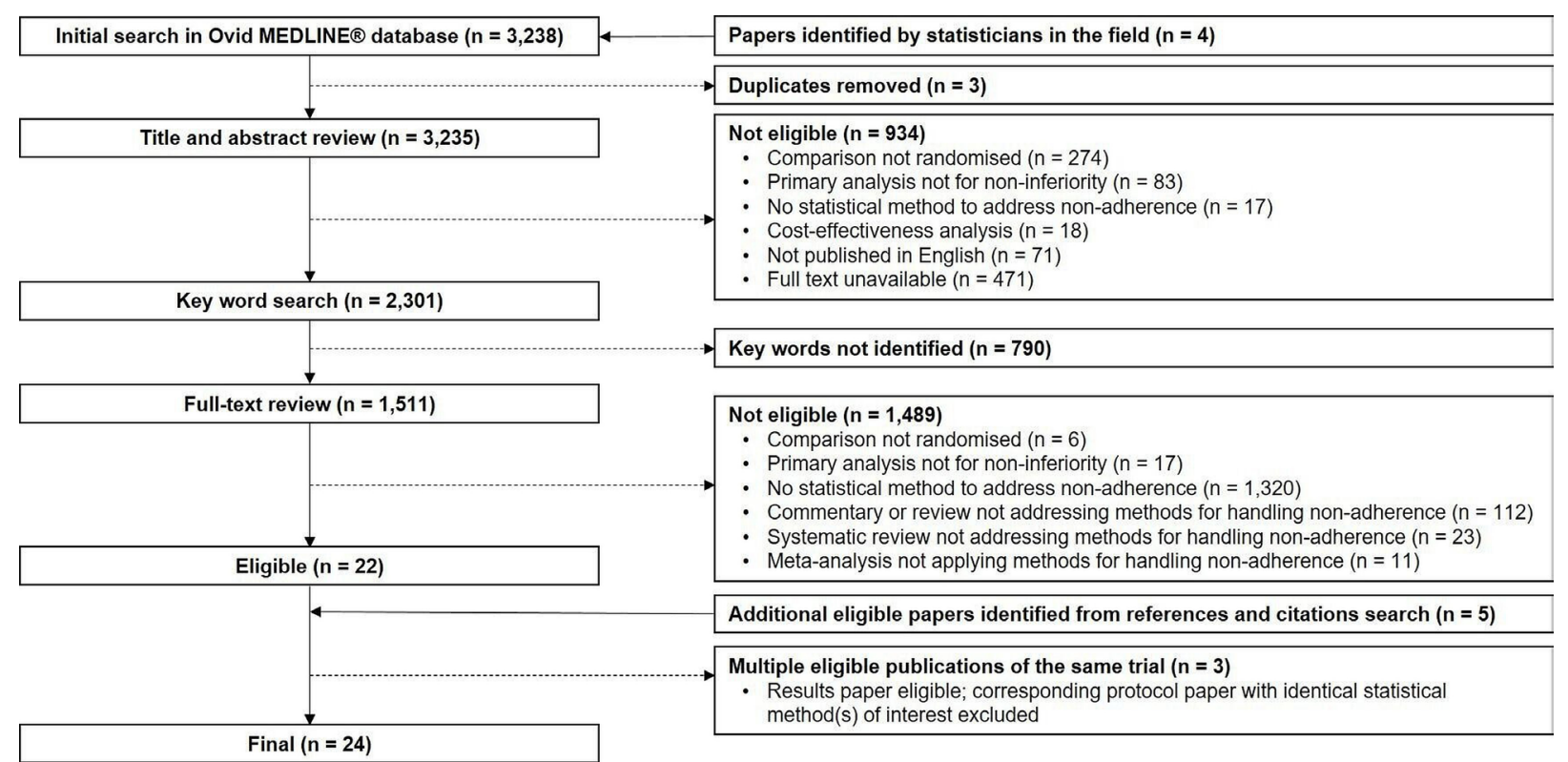

Figure 1 Flow chart showing the eligibility of papers reviewed (uploaded separately).

and G-estimation $(\mathrm{n}=2,8 \%)$, inverse-probability-oftreatment weighting $(n=2,8 \%)$ and the tipping point approach $(n=2,8 \%$, both in the same methodology paper). The other five techniques identified were all reported once. Further details of the methods reported more than once are provided in table 4 and online supplemental table A2. The techniques identified in the 17 protocols and results papers were more commonly specified as sensitivity analyses $(n=13,76 \%)$ than primary analyses $(n=3,18 \%)$ (one was unclear).

\section{Advantages and disadvantages of the statistical methods}

The advantages and disadvantages of the methods identified (as stated by the authors) are given in table 3. Advantages or disadvantages of the techniques used were stated in $8(33 \%)$ of the 24 papers included; 6 were methodological papers and 2 were results papers. No advantages or disadvantages were stated for 5 of the 11 methods identified.

\section{Impact of the statistical methods on non-inferiority conclusions}

Twelve of the 13 results papers (92\%) also included an alternative analysis of the same outcome (online supplemental table A3). All 12 performed an ITT or mITT analysis. In addition, some reported results from PP $(n=6,50 \%)$ or as-treated (AT; $n=2,17 \%)$ analyses. Non-inferiority conclusions from the alternate analyses were in agreement with those from the methods of interest on six occasions and could not be compared on six occasions (due to different measures of effect or the results not being provided in full). Five of the six analyses where the different methods were in agreement concluded non-inferiority of the experimental intervention versus the comparator. The remaining trial provided mixed findings regarding non-inferiority across the two different countries included, though the interpretation of this study appeared inconsistent with its design (a CI approach to determining non-inferiority was stated in the methods but not used).

\section{Statistical analysis plans}

Statistical analysis plans were requested for all 17 noninferiority trials where the protocol or results paper was included in the review, and obtained for nine of these trials.

\section{DISCUSSION}

To the best of our knowledge, this is the first systematic review undertaken to both identify statistical methods that adjust for the impact of non-adherence to interventions in randomised non-inferiority trials and also identify the frequency and consequences of their use. We found that few papers reported such methods (less than $2 \%$ of those reaching full-text review). This may be partly due to unfamiliarity with such techniques among trialists and statisticians as a result of the long lead time for statistical methodology to make its way into routine practice. The most common techniques identified were IV approaches, including observed adherence as a covariate within a regression model, and modelling adherence as a timevarying covariate in a time-to-event analysis. Overall, the number of trials implementing relevant statistical methods was too small to draw firm inferences about their impacts on non-inferiority conclusions. In six analyses where the results from methods of interest could be compared directly with those from an alternative analysis, conclusions regarding non-inferiority were consistent across the different approaches.

Almost half of the methods identified focus on estimating CACE (also known as the local average treatment effect (LATE)). This is the average effect of the 
Table 1 Characteristics of eligible analyses $(n=26)$

\begin{tabular}{|c|c|}
\hline Characteristics & n (\%) \\
\hline \multicolumn{2}{|l|}{ Type of publication $(n=24)$} \\
\hline Results & $13(54)$ \\
\hline Methodology & 7 (29) \\
\hline Protocol & $4(17)$ \\
\hline \multicolumn{2}{|l|}{ Year of publication $(n=24)$} \\
\hline $2006-2010$ & $5(21)$ \\
\hline 2011-2015 & $4(17)$ \\
\hline 2016-2020 & $15(63)$ \\
\hline \multicolumn{2}{|l|}{ Disease area or patient population } \\
\hline Mental health & $4(15)$ \\
\hline Appendicitis & $2(8)$ \\
\hline Cancer & $2(8)$ \\
\hline Respiratory infection/disease & $2(8)$ \\
\hline Ulcerative colitis & $2(8)$ \\
\hline Anaemia & $1(4)$ \\
\hline Ear infection & $1(4)$ \\
\hline General surgery patients & $1(4)$ \\
\hline Heart disease & $1(4)$ \\
\hline HIV & $1(4)$ \\
\hline Individuals receiving life-sustaining therapies & $1(4)$ \\
\hline Renal disease & $1(4)$ \\
\hline Smoking cessation & $1(4)$ \\
\hline Throat infection & $1(4)$ \\
\hline Urinary incontinence & $1(4)$ \\
\hline Simulation study & $4(15)$ \\
\hline
\end{tabular}

Unit of randomisation

\begin{tabular}{|c|c|}
\hline Individual & $19(73)$ \\
\hline Cluster & $3(12)$ \\
\hline Simulation study & $4(15)$ \\
\hline \multicolumn{2}{|l|}{ Type of experimental intervention } \\
\hline Drug & $9(35)$ \\
\hline Method of treatment delivery & $3(12)$ \\
\hline Additional patient examination & $2(8)$ \\
\hline Nutritional & $2(8)$ \\
\hline Surgical & $1(4)$ \\
\hline Simulation study & $4(15)$ \\
\hline Other & $5(19)$ \\
\hline \multicolumn{2}{|l|}{ Type of outcome } \\
\hline Binary & $13(50)$ \\
\hline Continuous & $8(31)$ \\
\hline Time to event & $4(15)$ \\
\hline Count & $1(4)$ \\
\hline Composite outcome & $3(12)$ \\
\hline
\end{tabular}

experimental intervention within the subpopulation of compliers. ${ }^{25}$ We argue that this is the natural estimation focus when attempting to account for non-adherence to interventions in the context of non-inferiority trials. This is because we want to be confident that there is non-inferiority among those who would comply with either intervention. By contrast, including participants who would not fully adhere to both interventions may bias estimation towards non-inferiority (in a similar way that, in the context of non-inferiority, ITT analyses may be biased towards non-inferiority under non-adherence). For similar reasons, we believe that the CACE is preferable to the population average treatment effect (ATE). Lastly, we note that when adjusting for observed adherence within a regression model or modelling adherence as a time-varying covariate in a time-to-event analysis, the target estimand is unclear.

The infrequent use of statistical methods for handling non-adherence seen in the current review has also been observed more generally in randomised controlled trials (RCTs). A review of 100 RCTs randomly selected from those published in 4 high-impact journals during 2008 found only 1 that attempted to account for non-adherence to interventions using a causal inference framework (in which inverse-probability-of-censoring weighting was applied). ${ }^{6}$ More recently, Mostazir et al conducted a review of statistical approaches for handling non-adherence to interventions in RCTs published between 1991 and 2015, which identified 88 analyses incorporating 9 different methods. ${ }^{28}$ IV methods were among the most common and accounted for almost one in four applications of suitable techniques. However, some of the other methods identified (including CACE analyses using maximumlikelihood estimation and adjusted treatment received models) were not captured in the current review focusing on non-inferiority trials. Similarly, we did not identify all 12 approaches included in a recent review of methodological papers containing statistical techniques for handling non-adherence to interventions in the context of time-to-event outcomes. ${ }^{29}$ This suggests that other relevant methods are available but either they are not suitable for comparing active interventions, as is often required in non-inferiority trials, or they may not have been applied within these studies. The three aforementioned reviews did not focus specifically on non-inferiority trials.

It is perhaps not surprising that IV approaches were the most common method identified in the current review, given that their assumptions are well suited to many double-blind trials, they can be applied across a range of trial designs, and they are relatively simple to implement in standard statistical software. ${ }^{30}$ IV methods use randomisation as the instrument in order to account for unmeasured confounders of the outcome and intervention received (ie, adherence). Their main assumptions are: (1) randomisation affects the outcome only through its influence on the intervention received (the exclusion restriction), (2) randomisation does not share common causes with the outcome (the exchangeability assumption), (3) randomisation causes some participants to receive their assigned intervention (the relevance assumption) and, in order to estimate CACE, (4) there are no participants who would always receive the opposite of their random allocation (the monotonicity assumption). ${ }^{23} 31$ In individually randomised trials, the exclusion restriction and 
monotonicity assumptions are typically satisfied by effective double blinding and/or use of objective outcomes, and the exchangeability assumption is usually valid since randomisation is expected to produce trial arms that are balanced with respect to prognostic factors.

When these assumptions hold, it is relatively straightforward to show that if we regress the intervention received (ie, adherence) on randomisation, and then use this model to predict each participant's adherence, these predictions are orthogonal (independent) of all adherence-outcome confounders. Therefore, if in a second step we regress the outcome on these predictions, we get an unconfounded estimate of the effect of adherence on outcome. It follows that, in contrast to techniques that involve inverse-probability weighting, when the above four IV assumptions hold, IV methods enable us to estimate CACE even in the presence of unmeasured confounding (although inclusion of measured confounders can improve precision). ${ }^{32} 33$ While IV methods may thus appear a panacea, as usual in statistics, there are no free lunches: a lack of precision and statistical power is often a challenge with IV techniques and methods used to adjust for non-adherence more generally. ${ }^{5303435}$

The two-stage least-squares (2SLS) regression approach sketched in the previous paragraph can be applied when the intervention is not all or nothing. Suppose that a non-inferiority trial is conducted to assess whether prescribing one dose of a medication per week is noninferior to prescribing two doses per week over the course of 4 weeks. For each participant, the monotonicity assumption requires that the potential number of doses taken would be lower if the participant was randomly assigned to receive one dose per week than if they were randomised to receive two doses per week. Assuming there are no covariates and the monotonicity assumption holds, it can be shown that the 2SLS estimator converges toward a weighted average of the causal effects of one unit increases in the intervention among compliers (individuals whose intervention intensity is affected by randomisation (the instrument)). ${ }^{36}{ }^{37}$ This is because the implicit effect of the 2SLS analysis is that values of the outcome at which there are more compliers get given greater weight.
A limitation of IV methods is that when interventions are administered at multiple timepoints, standard approaches are susceptible to time-varying confounding and selection bias. ${ }^{21}$ These biases occur when previous values of a covariate predict the current intervention received and the current value of the covariate predicts outcome. ${ }^{38}$ If the time-varying confounders are themselves affected by previous intervention received, so-called G-methods, such as inverse-probability weighting or G-estimation, are required to allow for the feedback loop occurring between the intervention received and confounders over time. ${ }^{21} 2439$ G-methods were seldom reported in the current review, perhaps because they can be more complex to implement than alternative approaches and also rely on assumptions which may be vulnerable to violations. When considering whether to apply an IV approach or a G-method, statisticians might consider whether the exclusion restriction and monotonicity assumptions are realistic given the context of the trial, and whether randomisation is a sufficiently strong instrument. Where outcomes are collected at multiple timepoints, inverse-probability weighting may be a more attractive approach if data on potential confounders are also collected throughout follow-up.

In order to estimate the effect of the experimental intervention in the absence of (full) protection by randomisation, additional assumptions must be made. Most of these are, by their nature, inherently untestable. Each of the statistical methods identified in the current review make slightly different assumptions in order to estimate the effects of interventions under full adherence, and hence each has a different method of estimation; both assumptions and estimation methods have associated advantages and disadvantages. Crucially, all of the methods require reliable information regarding adherence to the randomly assigned interventions, which is often challenging to measure, particularly for long-term therapies. ${ }^{40}$

Despite these limitations, it is our view that the methods identified have an important role in noninferiority trials with non-adherence to interventions and should be applied as sensitivity analyses

Table 2 Estimates of non-adherence to interventions reported in methodology and results papers, combined across trial arms unless reported $(n=13)$

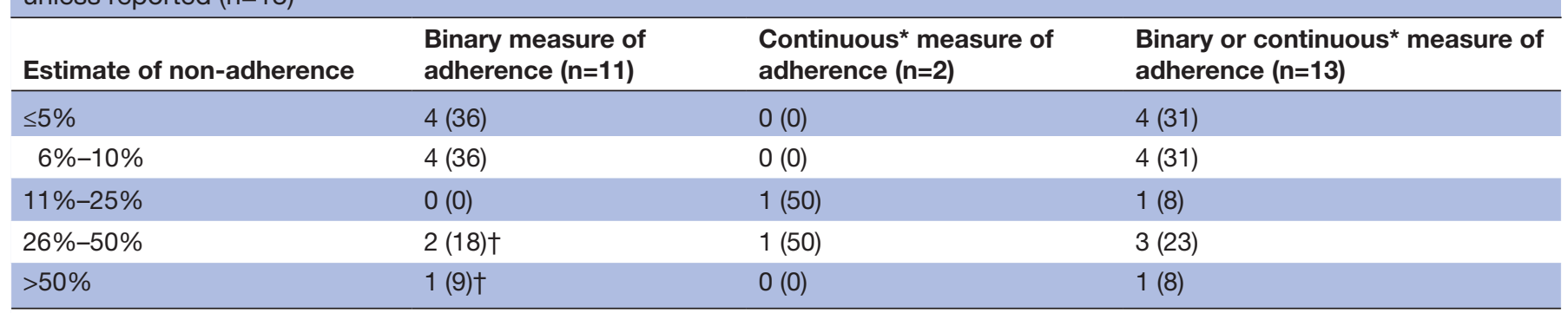

Data presented as $\mathrm{n}(\%)$.

${ }^{*}$ Mean level of non-adherence.

†Two papers provided an estimate of non-adherence in only one arm of the trial. 

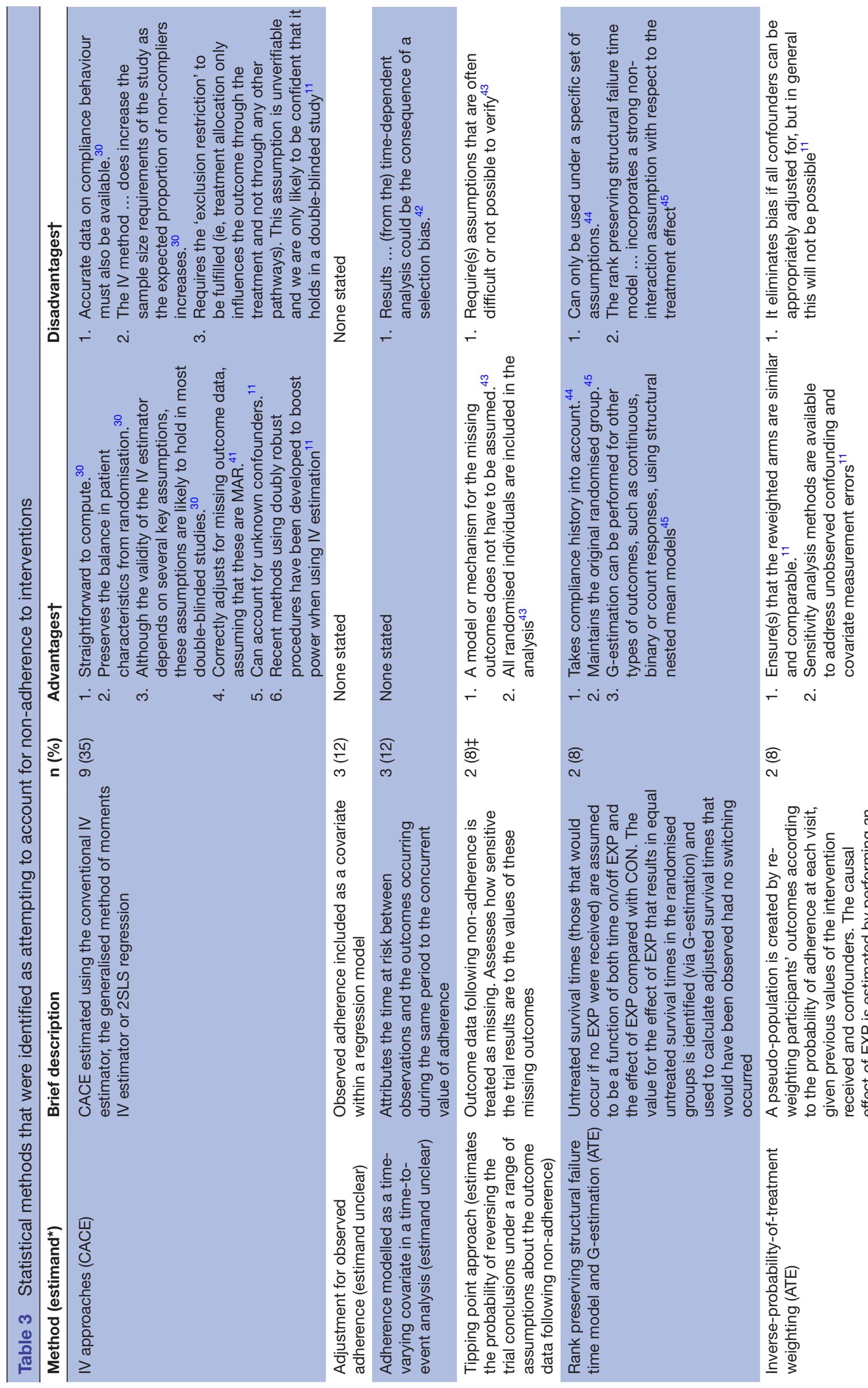

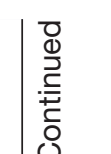

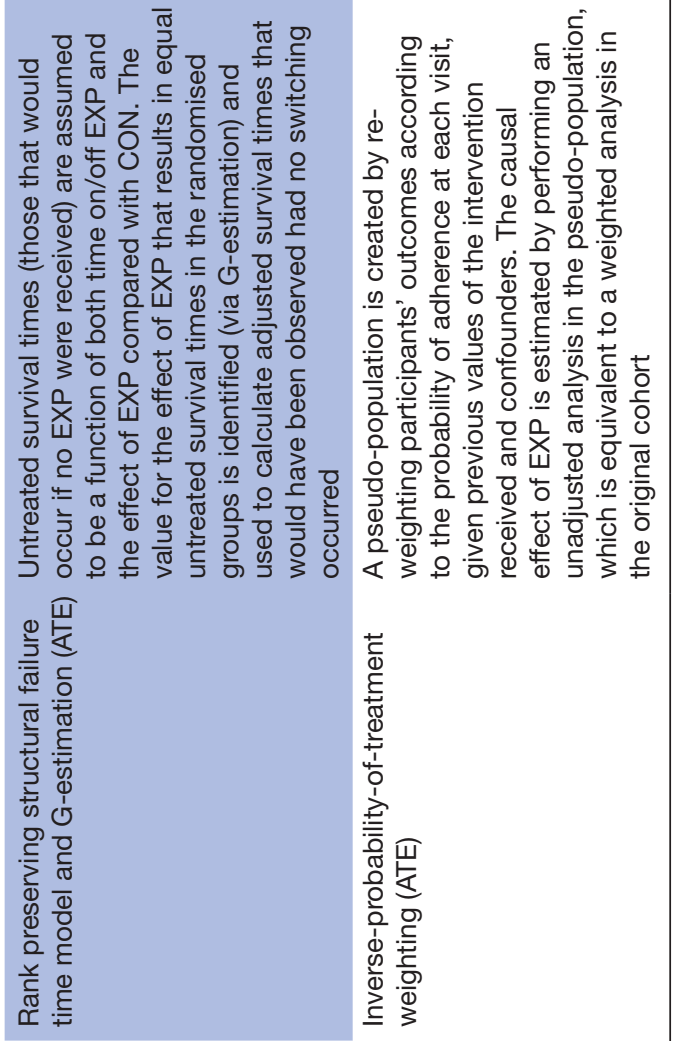




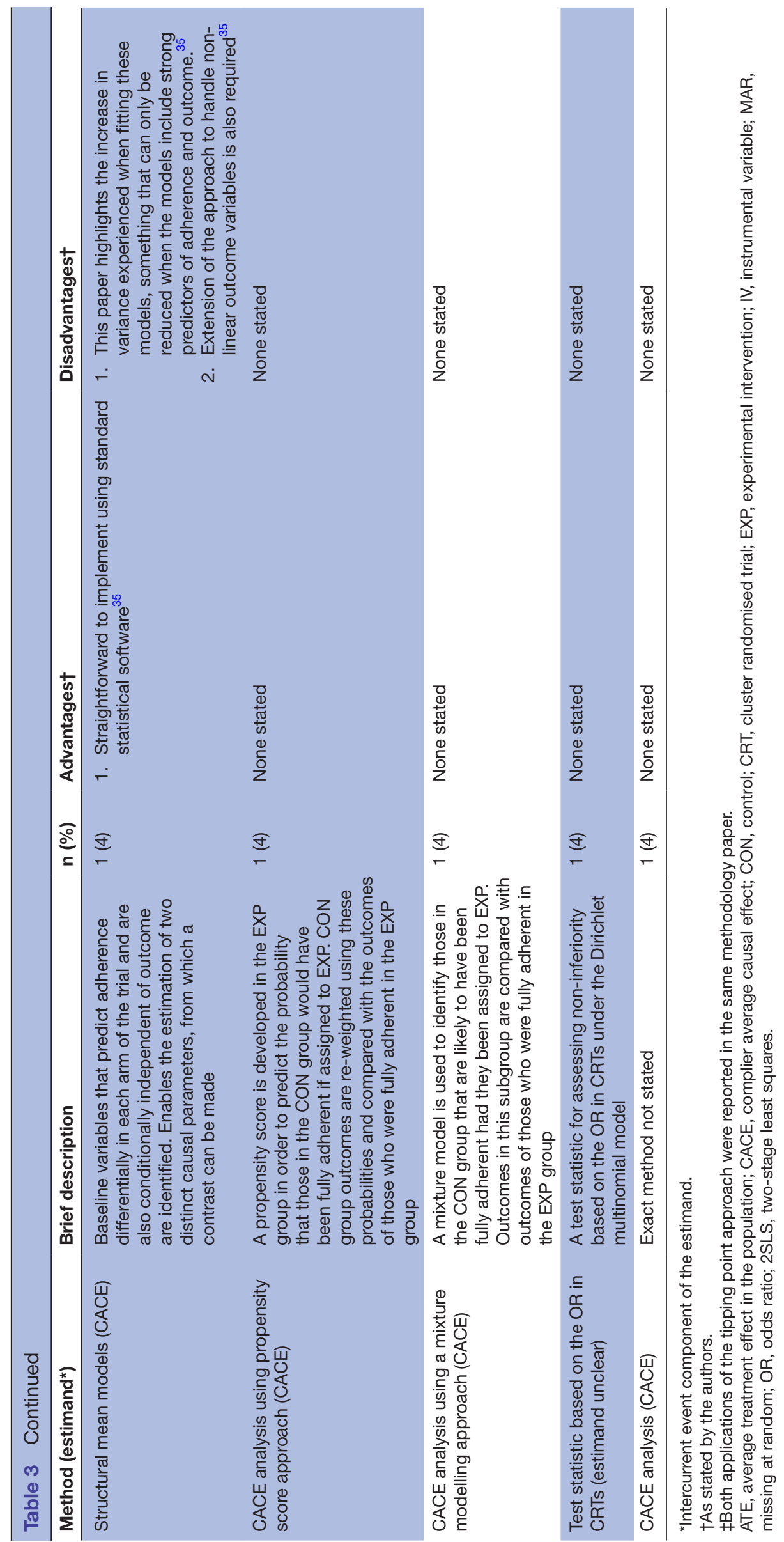




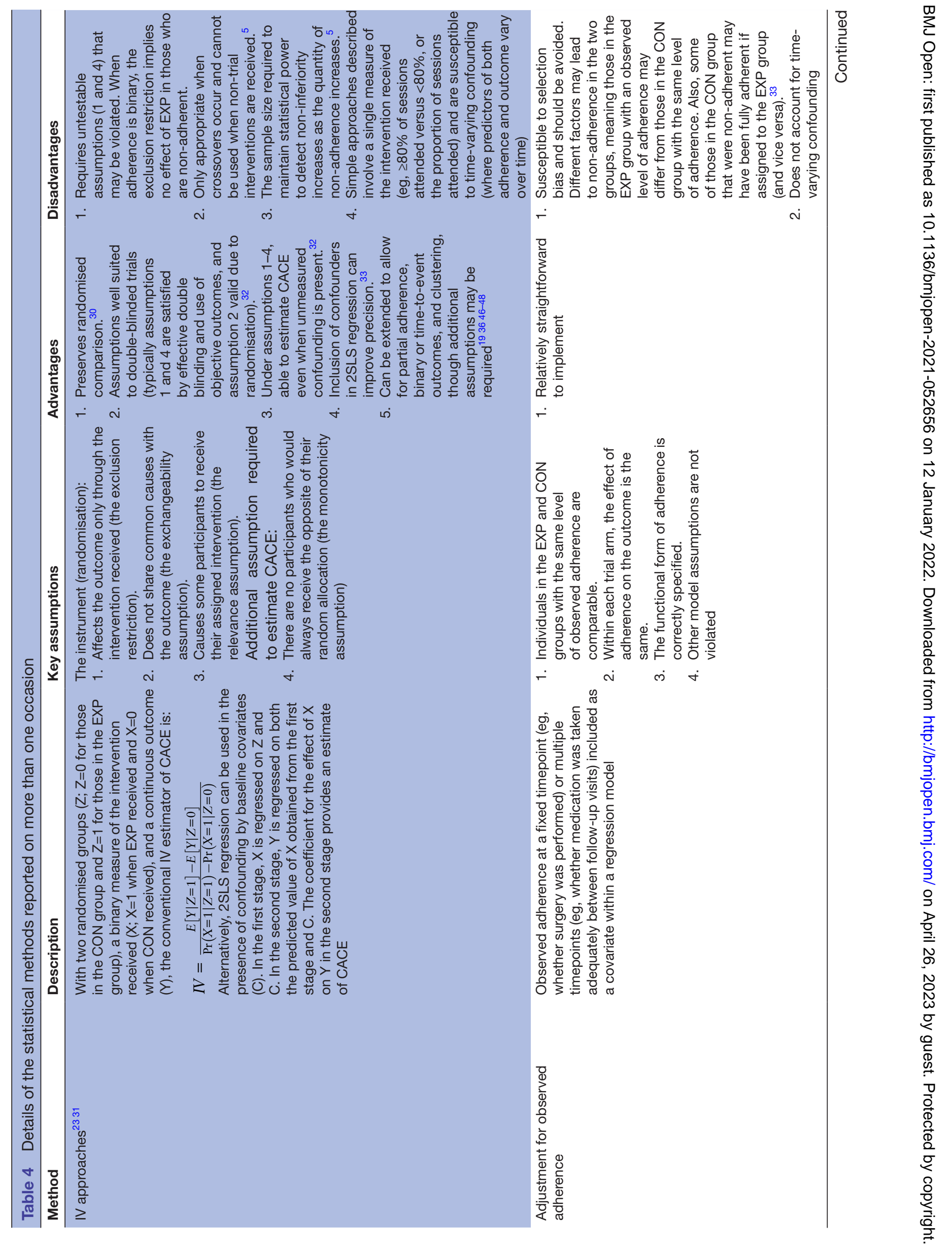




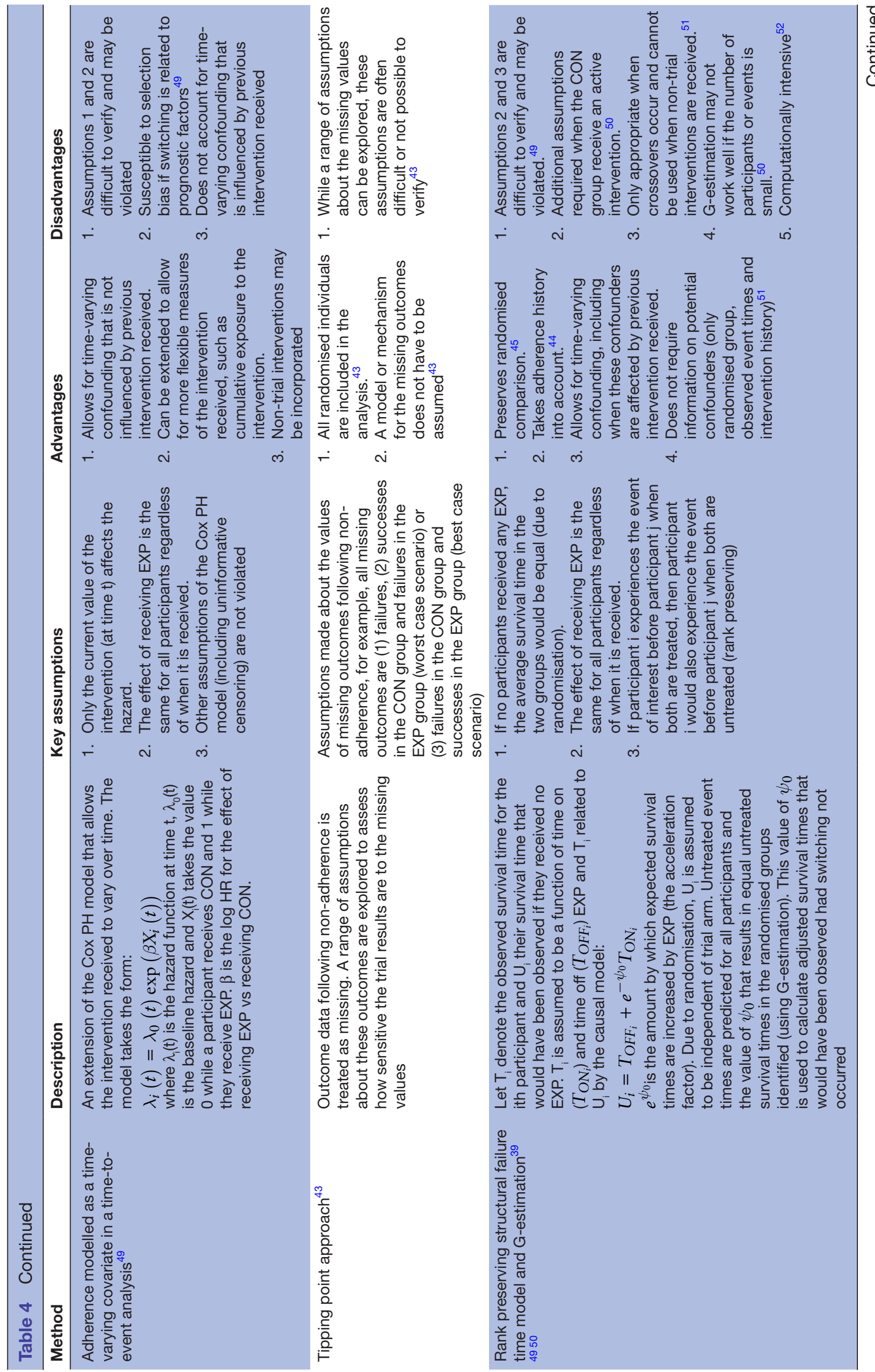



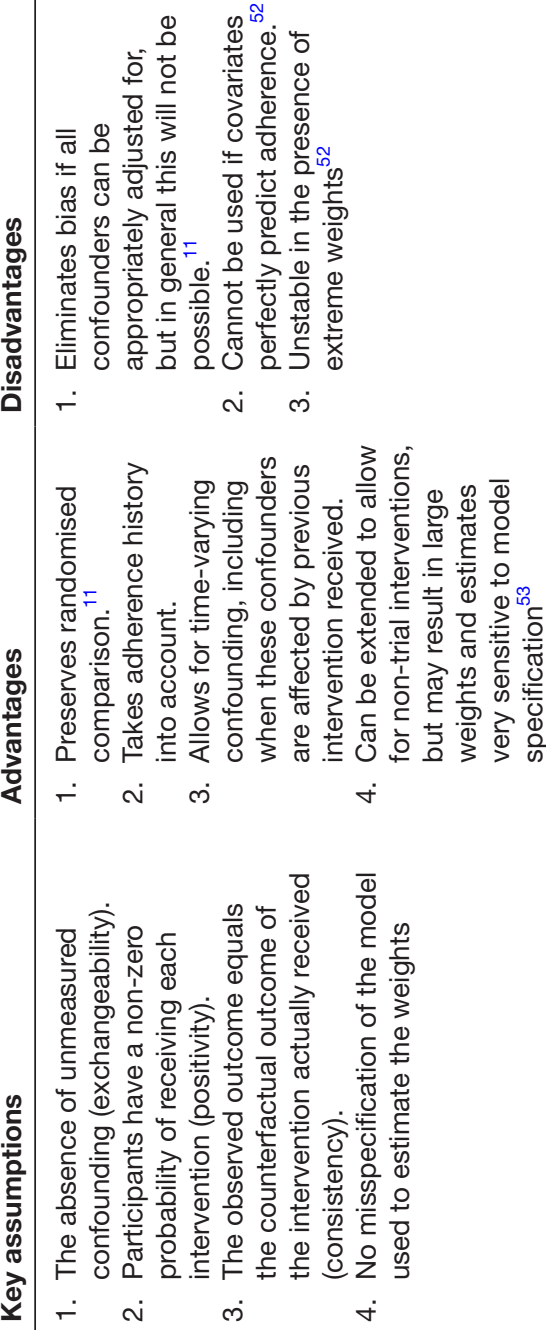

$\dot{r}$

$$
\text { i }
$$$$
\dot{m}
$$$$
\dot{\nabla}
$$

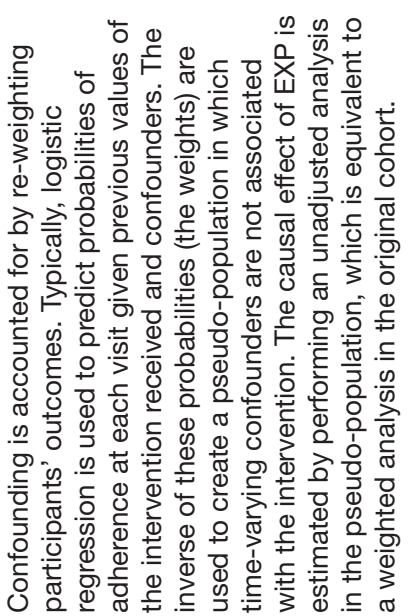

alongside other techniques (such as ITT, PP and AT analyses). Given that agreement between ITT and PP analyses cannot guarantee unbiased conclusions in these studies, those with non-trivial non-adherence should assess the sensitivity of trial results to different assumptions in order to guard against falsely claiming non-inferiority and accepting a worse intervention. Careful consideration needs to be given to the assumptions that are most plausible given the trial context and planned design, before selecting the appropriate statistical method to adjust for potential non-adherence. Relevant data needed to implement the chosen technique should then be collected as fully as possible. Clearly, the best approach to reducing the potential biases introduced by non-adherence to the interventions is to design trials that minimise such non-adherence. Future work should compare the performance of the methods identified under different non-adherence scenarios in non-inferiority trials to facilitate understanding of when they might be applied appropriately.

\section{Strengths and limitations}

This is the first systematic review (protocol published on PROSPERO) to identify statistical methods that attempt to account for the impact of non-adherence to interventions in randomised non-inferiority trials, quantify the use of such methods in these studies and examine their impact on non-inferiority conclusions. The review included publications from any year, journal or disease area and involved two authors agreeing the eligibility of each paper identified in the search. However, it has some limitations. First, the search for eligible papers had to be restricted to those containing terms related to adherence or statistical methods for handling nonadherence in the titles, abstracts and keywords. Publications applying suitable methods in sensitivity analyses may not have referred to the techniques within these fields and, if so, would not have been captured by the search. A wide range of search terms were used to try and mitigate this problem and, therefore, a large number of papers were reviewed. Second, only one database was searched and papers not published in English were excluded meaning it is possible that some eligible papers may not have been captured by the search. However, most major non-inferiority trials are likely to be published in English within one of the MEDLINE journals and, therefore, should have been captured. Third, one author performed the data extraction from eligible publications, though other authors were consulted where necessary. Finally, while statistical analysis plans were requested, these could not be obtained for eight of the trials included. For these studies, we cannot be sure that the details provided in the publications reviewed are accurate accounts of the planned analyses. 


\section{CONCLUSION}

In non-inferiority trials with non-adherence to interventions, ITT and PP analyses are often performed but may result in biased estimates of efficacy and, therefore, agreement between these approaches does not guarantee that conclusions regarding non-inferiority are unbiased. Statistical methods that attempt to account for the impact of non-adherence and thereby estimate the causal effects of interventions are available, but their use in non-inferiority trials remains extremely infrequent. It is our view that the methods identified should be applied more widely within sensitivity analyses of non-inferiority trials. In particular, those with non-trivial non-adherence should assess the sensitivity of trial results to different assumptions in order to guard against falsely claiming non-inferiority and accepting a worse intervention.

Contributors MD devised the review, reviewed the eligibility of papers identified, carried out data extraction and analysis, wrote the initial manuscript, and is responsible for the overall content as the guarantor. KF, JRC, JAT and DE commented on the review protocol, reviewed the eligibility of papers identified and assisted with writing the final manuscript. All authors provided final approval of the version to be published.

Funding JRC is supported by the Medical Research Council (MRC) Clinical Trials Unit (grant numbers: MC_UU_12023/21 and MC_UU_12023/29). JAT is jointly funded by the UK MRC and the UK Department for International Development (DFID) under the MRC/DFID Concordat agreement and is also part of the EDCTP2 programme supported by the European Union (grant number: MR/R010161/1).

Competing interests None declared.

Patient consent for publication Not applicable.

Ethics approval Ethical approval was not required because this review used publicly accessible documents.

Provenance and peer review Not commissioned; externally peer reviewed.

Data availability statement № data are available. No additional data are available.

Supplemental material This content has been supplied by the author(s). It has not been vetted by BMJ Publishing Group Limited (BMJ) and may not have been peer-reviewed. Any opinions or recommendations discussed are solely those of the author(s) and are not endorsed by BMJ. BMJ disclaims all liability and responsibility arising from any reliance placed on the content. Where the content includes any translated material, BMJ does not warrant the accuracy and reliability of the translations (including but not limited to local regulations, clinical guidelines, terminology, drug names and drug dosages), and is not responsible for any error and/or omissions arising from translation and adaptation or otherwise.

Open access This is an open access article distributed in accordance with the Creative Commons Attribution Non Commercial (CC BY-NC 4.0) license, which permits others to distribute, remix, adapt, build upon this work non-commercially, and license their derivative works on different terms, provided the original work is properly cited, appropriate credit is given, any changes made indicated, and the use is non-commercial. See: http://creativecommons.org/licenses/by-nc/4.0/.

\section{ORCID iDs}

Matthew Dodd http://orcid.org/0000-0002-6207-6604

Katherine Fielding http://orcid.org/0000-0002-6524-3754

James R Carpenter http://orcid.org/0000-0003-3890-6206

Jennifer A Thompson http://orcid.org/0000-0002-3068-3952

Diana Elbourne http://orcid.org/0000-0003-3044-4545

\section{REFERENCES}

1 Piaggio G, Elbourne DR, Altman DG, et al. Reporting of noninferiority and equivalence randomized trials: an extension of the CONSORT statement. JAMA 2006;295:1152-60.
2 Suda KJ, Hurley AM, McKibbin T, et al. Publication of noninferiority clinical trials: changes over a 20-year interval. Pharmacotherapy 2011;31:833-9.

3 Bikdeli B, Welsh JW, Akram Y, et al. Noninferiority designed cardiovascular trials in Highest-Impact journals. Circulation 2019;140:379-89.

4 Piaggio G, Elbourne DR, Pocock SJ, et al. Reporting of noninferiority and equivalence randomized trials: extension of the CONSORT 2010 statement. Jama 2012;308:2594-604.

5 Mo Y, Lim C, Watson JA, et al. Non-adherence in non-inferiority trials: pitfalls and recommendations. BMJ 2020;370:m2215.

6 Dodd S, White IR, Williamson P. Nonadherence to treatment protocol in published randomised controlled trials: a review. Trials 2012;13:84.

7 DiMatteo MR. Variations in patients' adherence to medical recommendations: a quantitative review of 50 years of research. Med Care 2004;42:200-9.

8 DiMatteo MR, Giordani PJ, Lepper HS, et al. Patient adherence and medical treatment outcomes: a meta-analysis. Med Care 2002:40:794-811.

9 Simpson SH, Eurich DT, Majumdar SR, et al. A meta-analysis of the association between adherence to drug therapy and mortality. BMJ 2006;333:15.

10 Ye C, Beyene J, Browne G, et al. Estimating treatment effects in randomised controlled trials with non-compliance: a simulation study. BMJ Open 2014;4:e005362.

11 Mo Y, Lim C, Mukaka M, et al. Statistical considerations in the design and analysis of non-inferiority trials with binary endpoints in the presence of non-adherence: a simulation study. Wellcome Open Res 2019;4:207.

12 Committee for Proprietary Medicinal Products. Points to consider on switching between superiority and non-inferiority. $\mathrm{Br} J \mathrm{Clin}$ Pharmacol 2001:52:223-8.

13 Rehal S, Morris TP, Fielding K, et al. Non-inferiority trials: are they inferior? A systematic review of reporting in major medical journals. BMJ Open 2016;6:e012594.

14 Matilde Sanchez M, Chen X. Choosing the analysis population in non-inferiority studies: per protocol or intent-to-treat. Stat Med 2006;25:1169-81.

15 Brittain E, Lin D. A comparison of intent-to-treat and per-protocol results in antibiotic non-inferiority trials. Stat Med 2005;24:1-10.

16 Clarke PS, Windmeijer F. Identification of causal effects on binary outcomes using structural mean models. Biostatistics 2010:11:756-70.

17 Anon. ICH Harmonised tripartite guideline. statistical principles for clinical trials. International Conference on harmonisation E9 expert Working group. Stat Med 1999;18:1905-42.

18 Gupta S. Intention-to-treat concept: a review. Perspect Clin Res 2011:2:109-12.

19 Nagelkerke N, Fidler V, Bernsen R, et al. Estimating treatment effects in randomized clinical trials in the presence of non-compliance. Stat Med 2000;19:1849-64.

20 Abraha I, Montedori A. Modified intention to treat reporting in randomised controlled trials: systematic review. BMJ 2010;340:c2697.

21 Hernán MA, Robins JM. Per-Protocol analyses of pragmatic trials. N Engl J Med 2017;377:1391-8.

22 DeMets DL. Statistical issues in interpreting clinical trials. $J$ Intern Med 2004:255:529-37.

23 Angrist JD, Imbens GW, Rubin DB. Identification of causal effects using instrumental variables. J Am Stat Assoc 1996;91:444-55.

24 Robins JM, Hernán MA, Brumback B. Marginal structural models and causal inference in epidemiology. Epidemiology 2000;11:550-60.

25 LHY Y, Little RJ. Inference for the Complier-Average causal effect from longitudinal data subject to Noncompliance and missing data, with application to a job training assessment for the unemployed. $J$ Am Stat Assoc 2001;96:1232-44.

26 Page MJ, Moher D, Bossuyt PM, et al. PRISMA 2020 explanation and elaboration: updated guidance and exemplars for reporting systematic reviews. BMJ 2021;372:n160.

27 Popay J, Roberts H, Sowden A, et al. Guidance on the conduct of narrative synthesis in systematic reviews: a product of the ESRC methods programme (version I). Lancaster, UK: University of Lancaster, 2006.

28 Mostazir M, Taylor RS, Henley W, et al. An overview of statistical methods for handling nonadherence to intervention protocol in randomized control trials: a methodological review. J Clin Epidemiol 2019;108:121-31.

29 Alshreef A, Latimer N, Tappenden P, et al. Statistical methods for adjusting estimates of treatment effectiveness for patient nonadherence in the context of Time-to-Event outcomes and health 
technology assessment: a systematic review of methodological papers. Med Decis Making 2019;39:910-25.

$30 \mathrm{Kim}$ MY. Using the instrumental variables estimator to analyze noninferiority trials with noncompliance. J Biopharm Stat 2010;20:745-58

31 Dunn G, Maracy M, Tomenson B. Estimating treatment effects from randomized clinical trials with noncompliance and loss to follow-up: the role of instrumental variable methods. Stat Methods Med Res 2005;14:369-95.

32 Lousdal ML. An introduction to instrumental variable assumptions, validation and estimation. Emerg Themes Epidemiol 2018;15:1.

33 Stuart EA, Perry DF, Le H-N, et al. Estimating intervention effects of prevention programs: accounting for noncompliance. Prev Sci 2008;9:288-98.

34 Davies NM, Smith GD, Windmeijer F, et al. Issues in the reporting and conduct of instrumental variable studies. Epidemiology 2013;24:363-9.

35 Gillespie D, Farewell D, Barrett-Lee P, et al. The use of randomisation-based efficacy estimators in non-inferiority trials. Trials 2017;18:117.

36 Angrist JD, Imbens GW. Two-Stage least squares estimation of average causal effects in models with variable treatment intensity. $J$ Am Stat Assoc 1995;90:431-42.

37 Baiocchi M, Cheng J, Small DS. Instrumental variable methods for causal inference. Stat Med 2014;33:2297-340.

38 Daniel RM, Cousens SN, De Stavola BL, et al. Methods for dealing with time-dependent confounding. Stat Med 2013;32:1584-618.

39 Hernán MA, Cole SR, Margolick J, et al. Structural accelerated failure time models for survival analysis in studies with time-varying treatments. Pharmacoepidemiol Drug Saf 2005;14:477-91.

40 Lam WY, Fresco P. Medication adherence measures: an overview. Biomed Res Int 2015;2015:1-12.

41 Kitchener HC, Dunn G, Lawton V, et al. Laparoscopic versus open colposuspension--results of a prospective randomised controlled trial. BJOG 2006;113:1007-13.

42 Brunori G, Viola BF, Parrinello G, et al. Efficacy and safety of a very-low-protein diet when postponing dialysis in the elderly: a prospective randomized multicenter controlled study. Am J Kidney Dis 2007;49:569-80.
43 Kim M, Wang C, Xue X. Assessing the influence of treatment nonadherence on noninferiority trials using the tipping point approach. Stat Med 2019;38:650-9.

$44 \mathrm{Wu}$ Y, Zhao L, Hou Y, et al. Correcting for non-compliance in randomized non-inferiority trials with active and placebo control using structural models. Stat Med 2015;34:950-65.

45 Matsuyama Y. A comparison of the results of intent-to-treat, per-protocol, and g-estimation in the presence of non-random treatment changes in a time-to-event non-inferiority trial. Stat Med 2010;29:2107-16.

46 Palmer TM, Thompson JR, Tobin MD, et al. Adjusting for bias and unmeasured confounding in Mendelian randomization studies with binary responses. Int J Epidemiol 2008;37:1161-8.

47 Tchetgen Tchetgen EJ, Walter S, Vansteelandt S, et al. Instrumental variable estimation in a survival context. Epidemiology 2015;26:402-10.

48 Agbla SC, De Stavola B, DiazOrdaz K. Estimating cluster-level local average treatment effects in cluster randomised trials with nonadherence. Stat Methods Med Res 2020;29:911-33.

49 Morden JP, Lambert PC, Latimer N, et al. Assessing methods for dealing with treatment switching in randomised controlled trials: a simulation study. BMC Med Res Methodol 2011;11:4.

50 Latimer NR, Henshall C, Siebert U, et al. Treatment switching: statistical and decision-making challenges and approaches. Int $J$ Technol Assess Health Care 2016;32:160-6.

51 Allison A, White IR, Bond S. rpsftm: an R package for RANK preserving structural failure time models. $R J$ 2017;9:342-53.

52 Mansournia MA, Etminan M, Danaei G, et al. Handling time varying confounding in observational research. BMJ 2017;359:j4587.

53 Ellis AR, Brookhart MA. Approaches to inverse-probability-oftreatment--weighted estimation with concurrent treatments. J Clin Epidemiol 2013;66:S51-6.

54 Austin PC, Stuart EA. Moving towards best practice when using inverse probability of treatment weighting (IPTW) using the propensity score to estimate causal treatment effects in observational studies. Stat Med 2015;34:3661-79. 